PROCEEDINGS OF THE

AMERICAN MATHEMATICAL SOCIETY

Volume 131, Number 7, Pages 2137-2144

S 0002-9939(02)06766-7

Article electronically published on October 24, 2002

\title{
SECOND ORDER SINGULAR PERIODIC PROBLEMS IN THE PRESENCE OF DRY FRICTION
}

\author{
A. CABADA AND L. SANCHEZ
}

(Communicated by Carmen C. Chicone)

\begin{abstract}
We prove, via an approach by ordinary differential equations, the existence of oscillations for second order inclusions with restoring terms with singularities both of repulsive and attractive type and with a dry friction term.
\end{abstract}

\section{INTRODUCTION}

The existence and properties of periodic solutions for differential equations of type

$$
u^{\prime \prime}+g(u)+f\left(u^{\prime}\right)+\mu \operatorname{sign} u^{\prime}=\varphi(t),
$$

where $f, g$ are continuous, $\varphi$ is periodic and $\mu>0$, have attracted the attention of several authors in the last decade. Equation (1) models a one-dimensional mechanical system subjected to dry friction and periodic forcing. Dry friction arises when two rigid bodies interact in such a way that contact phases are interrupted by slip phases. In one of its simplest forms, this non-smooth mechanical effect can be modelled by Coulomb's law, according to which the term $\mu$ sign $u^{\prime}$ has been inserted. Additional viscous damping may be represented by $f\left(u^{\prime}\right)$, and $g(u)$ is a restoring force depending on position. We refer the reader to the survey by Kunze 9] for a wealth of information on this kind of problem.

Existence, nonexistence and properties of periodic solutions of such systems have been considered by Deimling [4, Deimling and Szilágyi [5] and Bothe 2]. In [4] problems of existence and uniqueness are discussed under the assumption that $f=0$ and $g$ is linear. In particular an existence result for resonance at the first nonzero eigenvalue is obtained. (See also section 2.)

The case when $f$ and $g$ are both linear and (1) may contain an extra bounded perturbation has been considered by Senkyrik [12, Senkyrik and Guenther [13] and more recently by Bothe [2]; in the latter article new interesting results are obtained for the case of $u$-dependent $\mu=\mu(u)$.

In all the papers mentioned above equation (1) is viewed as a differential inclusion where sign is interpreted in the usual way as a maximal monotone graph. The

Received by the editors October 23, 2001 and, in revised form, February 18, 2002.

2000 Mathematics Subject Classification. Primary 34B15, 34C25.

The first author's research was supported by D.G.I. project BFM 2001-3884-C02-01 and Acción integrada Hispano - Lusa HP199-0026.

The second author's research was supported by Fundação para a Ciência e a Tecnologia and Acção Integrada Luso-Espanhola E/93-00. 
techniques used by these authors naturally involve multi-valued mappings and the theory of differential inclusions. In [7] results about (1) are given as corollaries of general existence theorems that use the concept of solution in the sense of Filipov's [6].

In this note our purpose is twofold. We address the problem of existence of periodic solutions to (1) in a singular setting, namely when $g(u)$ is defined for $u>0$ only and becomes infinite near $u=0$. In other words, we consider the onedimensional motion of a particle in a singular force field (e.g. due to an attractive or repulsive electrical source) and affected by periodic forcing and dry friction. On the other hand, although we look at (1) as a differential inclusion, we exploit a hint of Deimling [4] and show that a simple approximation procedure can be carried out, with resource to well known tools to solve nonlinear problems in the field of ordinary differential equations, to obtain in the end a solution in the sense of inclusions.

The study of periodic solutions under one-dimensional singular fields was initiated by Lazer and Solimini [10] for conservative systems. Analogous results for the case where dissipation is present were obtained in [8] and more recently in [1].

We shall consider both attractive and repulsive forces and we take $f=0$, so that damping is restricted to dry friction. In the attractive case we allow $g$ to grow linearly below a certain slope related to the period $T$ as has already been done, e.g. in [1], [15. In the repulsive case we combine the use of upper and lower solutions with a device of Mawhin [11] concerning problems where the nonlinearities depend only on the derivative.

In order to show how our (simple) approximation procedure works we give in section 2 a slight improvement of a result of Deimling [4 for a nonsingular problem; in its proof we present the main idea to be used in the remaining proofs.

Finally it should be noted that, as in other papers already mentioned in connection with the dry friction problem, e.g. [2, 4], there are simple conditions that one can formulate in order to prevent (1) from possessing constant solutions, so that in such cases our results do not reduce to trivial statements.

The authors wish to thank the referee for careful reading of the manuscript and for valuable suggestions to improve its presentation.

Notation. When we deal with functions $u$ defined in a given interval $[0, T]$ we denote by $\|u\|_{X}$ the standard norm of $u$ in the functional space $X$ and by $\|u\|_{p}(p \geq 1)$ the $L^{p}(0, T)$-norm of $u$.

\section{Approximation PROCEDURE AND PERIODIC SOLUTION OF A RESONANT PROBLEM}

In this section we consider the following periodic problem:

$$
u^{\prime \prime}+\mu(u) S\left(u^{\prime}\right)+u=\varphi(t), \quad u(0)=u(2 \pi), \quad u^{\prime}(0)=u^{\prime}(2 \pi),
$$

where

(A) $S$ is a function defined, bounded and continuous in $\mathbb{R} \backslash 0$ with a jump discontinuity at $0, S\left(0^{-}\right)<S\left(0^{+}\right)$, and

$$
\alpha:=\limsup _{z \rightarrow-\infty} S(z)<0<\beta:=\liminf _{z \rightarrow+\infty} S(z),
$$

(B) $\varphi$ is continuous, $2 \pi$-periodic, and

(C) $\mu$ is a continuous function in $\mathbb{R}$ such that there exist numbers $\mu_{0}$ and $\mu_{1}$ such that $0<\mu_{0} \leq \mu(u) \leq \mu_{1}, \forall u \in \mathbb{R}$. 
By a solution of (2.1) we mean a $2 \pi$-periodic function $u \in W^{2,1}(0,2 \pi)$ such that there exists $w \in L^{\infty}(0,2 \pi)$ satisfying $S\left(0^{-}\right) \leq w(t) \leq S\left(0^{+}\right)$a.e. in $B=\left\{t: u^{\prime}(t)=\right.$ $0\}, w(t)=S\left(u^{\prime}(t)\right)$ a.e. in $A:=\left\{t: u^{\prime}(t) \neq 0\right\}$ and

$$
u^{\prime \prime}+\mu(u) w(t)+u=\varphi(t), \quad \text { a.e., } \quad u(0)=u(2 \pi), \quad u^{\prime}(0)=u^{\prime}(2 \pi) .
$$

The following theorem illustrates how we solve problems like (2.1) where a similar definition of solution is applicable. It may be related to results of Deimling [4] and Bothe [2].

Since (2.1) contains in the left-hand side a noninvertible operator, we shall use decompositions of functional spaces into its kernel and a complementary subspace: we set

$$
L^{2}(0,2 \pi)=E_{1} \oplus E_{2}
$$

where $E_{1}:=\operatorname{span}\{\cos t, \sin t\}$ and $\oplus$ denotes orthogonal direct sum. Accordingly, we split each $u \in L^{2}(0,2 \pi)$ as

$$
u=u_{1}+u_{2}, \quad u_{i} \in E_{i}, \quad i=1,2 .
$$

Theorem 1. Suppose that (A), (B), (C) hold and $\left\|\varphi_{1}\right\|_{\infty}<2 \mu_{0}(\beta-\alpha) / \pi$. Then the problem (2.1) has at least one solution.

Proof. 1st step: approximated problem. Let $P_{i}$ denote the orthogonal projection onto $\overline{E_{i}}$. For each small $\epsilon>0$ define $S_{\epsilon}$ as the continuous function such that $S_{\epsilon}(z)=S(z)$ if $|z| \geq \epsilon$ and $S_{\epsilon}$ is linear in $[-\epsilon, \epsilon]$. Now consider the system

$$
\left\{\begin{array}{l}
u_{2}^{\prime \prime}+u_{2}+\lambda P_{2} \mu(u) S_{\epsilon}\left(u_{1}^{\prime}+\lambda u_{2}^{\prime}\right)=\varphi_{2} \\
P_{1} \mu\left(u_{1}+\lambda u_{2}\right) S_{\epsilon}\left(u_{1}^{\prime}+\lambda u_{2}^{\prime}\right)=\varphi_{1}
\end{array}\right.
$$

where $\lambda \in[0,1]$. Clearly, when $\lambda=1$, this is equivalent to the approximated problem for an ordinary differential equation

$$
u^{\prime \prime}+\mu(u) S_{\epsilon}\left(u^{\prime}\right)+u=\varphi(t), \quad u(0)=u(2 \pi), \quad u^{\prime}(0)=u^{\prime}(2 \pi) .
$$

To prove that (2.3) has a solution we show that (a) the solutions of (2.2) are a priori bounded independently of $\lambda$ and (b) noting that system (2.2) may be written as $u-T(\lambda, u)=0$, where $T$ is compact in $[0,1] \times C^{1}[0,2 \pi]$, the Leray-Schauder degree of $u \mapsto u-T(0, u)$ in a large ball is $\neq 0$.

Proof of (a): First we note that as a consequence of (A)-(C) fixing $\alpha<\alpha^{*}<$ $0<\beta^{*}<\beta$ there is a constant $C$ independent of $\epsilon$ so that for any function $v \in$ $W^{1,1}(0,2 \pi)$ one has

$$
\int_{0}^{2 \pi} \mu(v) S_{\epsilon}\left(v^{\prime}\right) v^{\prime} d t \geq \mu_{0}\left(\beta^{*}\left\|v^{\prime+}\right\|_{1}-\alpha^{*}\left\|v^{\prime-}\right\|_{1}\right)-C .
$$

Now from the first equation (2.2), our assumptions and the fact that the linear map $u_{2} \mapsto u_{2}^{\prime \prime}+u_{2}$ is invertible in $E_{2}$, it follows that there is $C^{\prime}>0$ independent of $\lambda$ and $\epsilon$ so that for any possible solution,

$$
\left\|u_{2}\right\|_{C^{2}} \leq C^{\prime}
$$

Let us take the second equation, multiply it by $u_{1}^{\prime}$ and integrate in $[0,2 \pi]$. Note that if $z \in E_{1}$, then $\int_{0}^{2 \pi} z^{+}=\int_{0}^{2 \pi} z^{-}$and $z^{\prime} \in E_{1}$ as well. Therefore, using (2.4) and the boundedness of $\mu$ and $S$, we obtain with another such constant $D$

$$
\mu_{0}\left(\beta^{*}-\alpha^{*}\right) \frac{\left\|u_{1}^{\prime}\right\|_{1}}{2} \leq D\left\|u_{2}^{\prime}\right\|_{1}+\frac{\pi}{4}\left\|\varphi_{1}\right\|_{\infty}\left\|u_{1}^{\prime}\right\|_{1}+C .
$$

Since we can assume that $\left\|\varphi_{1}\right\|_{\infty}<2 \mu_{0}\left(\beta^{*}-\alpha^{*}\right) / \pi$, the boundedness of $u$ follows. 
Proof of (b): Note that, by well known facts of degree theory (see e.g. 3 for details), when $\lambda=0$ the calculation of the degree for the compact perturbation of the identity involved in (2.2) reduces to that of the 2-dimensional operator $F\left(u_{1}\right):=$ $P_{1} \mu\left(u_{1}\right) S_{\epsilon}\left(u_{1}^{\prime}\right)-\varphi_{1}$ defined in $E_{1}$. Using the natural inner product (denoted ·) in this space, multiplying by $u_{1}^{\prime}$ and integrating as above it turns out that for all $u_{1} \in$ $E_{1}$ on the boundary of a sufficiently large ball $B$ one has $F\left(u_{1}\right) \cdot d\left(u_{1}\right)>0$, where $d$ is the differentiation operator. Since $d$ is an isomorphism we have $|\operatorname{deg}(F, B)|=1$.

2nd step: passing to the limit. As a consequence of the estimates in the 1st step, the family of problems (2.3) has solutions $u_{\epsilon}$ that are uniformly bounded with respect to $\epsilon$. Hence, by elementary compactness arguments, along a sequence $\epsilon \rightarrow 0$ we may assume that for some functions $u, w$

$$
u_{\epsilon} \rightarrow u \text { in } W^{2, \infty}(0,2 \pi) \text { and } S_{\epsilon}\left(u_{\epsilon}^{\prime}\right) \rightarrow w \text { in } L^{\infty}(0,2 \pi)-w e a k^{*} .
$$

Consider the sets $A_{\delta}:=\left\{t:\left|u^{\prime}(t)\right| \geq \delta\right\}(\delta>0)$ and $B:=\left\{t: u^{\prime}(t)=0\right\}$. Since $u_{\epsilon}^{\prime} \rightarrow u^{\prime}$ uniformly, our definition of $S_{\epsilon}$ implies that for $\epsilon$ sufficiently small $S_{\epsilon}\left(u_{\epsilon}^{\prime}\right)=S\left(u_{\epsilon}^{\prime}\right)$ in $A_{\delta}$ and we conclude $w(t)=S\left(u^{\prime}(t)\right)$ a.e. in $A_{\delta}$; the arbitrariness of $\delta$ shows that the same is true a.e. in $A$. Given $\delta>0$, there exist $\eta>0, \epsilon_{0}>0$ such that

$$
S\left(0^{-}\right)-\delta \leq S_{\epsilon}(z) \leq S\left(0^{+}\right)+\delta \text { whenever }|z| \leq \eta \quad \text { and } \quad \epsilon \leq \epsilon_{0} .
$$

Again using the uniform convergence of $u_{\epsilon}^{\prime}$ in the set $B_{\eta}:=\left\{t:\left|u^{\prime}(t)\right| \leq \eta / 2\right\}$, we see that the restriction of $S_{\epsilon}\left(u_{\epsilon}^{\prime}\right)$ to this set takes values in $\left[S\left(0^{-}\right)-\delta, S\left(0^{+}\right)+\delta\right]$ for $\epsilon \leq \epsilon_{0}$. By weak-star convergence, the same is true for $w$ a.e. Considering a sequence $\delta \rightarrow 0$, we may assume that the corresponding $\eta \rightarrow 0$ as well. We can write $B$ as a countable intersection of the $B_{\eta}$ 's and it is straightforward to conclude that $w(t) \in\left[S\left(0^{-}\right), S\left(0^{+}\right)\right]$a.e. in $B$. This completes the proof.

\section{RePUlsive FORCES}

In this and in the following section we consider the problem

$$
u^{\prime \prime}+H\left(u^{\prime}\right)+g(u)=\varphi(t), \quad u(0)=u(T), \quad u^{\prime}(0)=u^{\prime}(T)
$$

$(T>0)$. We decompose the continuous, $T$-periodic function $\varphi(t)=\bar{\varphi}+\tilde{\varphi}(t)$ where $\bar{\varphi}$ is the mean value of $\varphi$ in $[0, T]$. We assume

(N) $H$ is a continuous function in $\mathbb{R} \backslash 0$ so that $H(z) z \geq 0$ if $z \neq 0, H\left(0^{-}\right)<$ $0<H\left(0^{+}\right)$and normalized so that

$$
\inf H=-1, \quad \sup H=1 .
$$

In our first result $g(u)$ is bounded above:

Theorem 2. Let $g:] 0,+\infty[\rightarrow \mathbb{R}$ be a continuous function such that

(i) either there exists $R \in \mathbb{R}$ such that $g(R)>\varphi(t)+1, \forall t \in \mathbb{R}$, or there exists an interval $J$ with $g(u)>\bar{\varphi}+1 \forall u \in J$ and the length of $J$ is $>C_{1} T^{3 / 2} / 12$ where $C_{1}=1+\sup (g-\bar{\varphi})+\sup |\tilde{\varphi}|$

(ii) $\int_{0}^{1} g(u) d u=-\infty$.

Then the problem (3.1) admits at least one solution.

Proof. For each $\epsilon>0$ define a continuous function $H_{\epsilon}: \mathbb{R} \rightarrow \mathbb{R}$ by

$$
H_{\epsilon}(x)= \begin{cases}H(x) & \text { if }|x| \geq \epsilon \\ H(\epsilon) x / \epsilon & \text { if } 0 \leq x \leq \epsilon \\ -H(-\epsilon) x / \epsilon & \text { if }-\epsilon \leq x \leq 0\end{cases}
$$


and consider the approximated problem

$$
u^{\prime \prime}+H_{\epsilon}\left(u^{\prime}\right)+g(u)=\varphi(t) .
$$

We shall show that (3.3) has a $T$-periodic solution $u_{\epsilon}$ and we shall obtain bounds for this solution that are independent of $\epsilon$. Once this is done we extract a converging subsequence as $\epsilon \rightarrow 0$ and we obtain a solution to (3.1) in the same way as has been done in the preceding section. To this end, let us consider the homotopy

(3.4) $u^{\prime \prime}+H_{\epsilon}\left(u^{\prime}\right)+g(u, \lambda)=\bar{\varphi}+\lambda \tilde{\varphi}(t), u(0)=u(T), u^{\prime}(0)=u^{\prime}(T), \quad 0 \leq \lambda \leq 1$.

Here we define

$$
g(u, \lambda)=(1-\lambda) g_{0}(u)+\lambda g(u),
$$

where $\left.g_{0}:\right] 0,+\infty\left[\rightarrow \mathbb{R}\right.$ is chosen as a strictly increasing $C^{1}$ function, with properties (i) (with the same $R$ or $J$ ) and (ii), such that $\sup g_{0}-\bar{\varphi}=\sup g-\bar{\varphi}$.

Fix $r>0$ such that $g(r), g_{0}(r)<\inf \varphi-1$. Let $R$ be given by (i) and, in case the second condition there described holds, let $R$ be the left endpoint of $J$. We consider the set $S$ of solutions $u$ of (3.4) for some $\epsilon$ and $\lambda$ such that max $u>r$ and $\min u<R$.

Claim. There exist $\alpha, \beta>0$ independent of $\epsilon$ and $\lambda$ such that for every $u \in S$ we have $u(t)>\alpha \forall t \in[0, T]$ and $\|u\|_{C^{1}[0, T]}<\beta$.

Proof of the Claim. For any given $u \in S$ let $m:=\min u$. Multiplying (3.4) by $u-m$ and integrating in $[0, T]$ we obtain

$$
\int_{0}^{T} u^{\prime}(t)^{2} d t \leq C_{1} \int_{0}^{T}(u(t)-m) d t .
$$

Now, for functions $w \in H_{0}^{1}(0, T)$ the inequality

$$
\left|\int_{0}^{T} w(t) d t\right| \leq \frac{T^{3 / 2}}{12}\left(\int_{0}^{T} w^{\prime}(t)^{2} d t\right)^{1 / 2}
$$

holds. Since periodic functions can be translated without affecting their integrals over a period, we have from (3.5)

$$
\left(\int_{0}^{T} u^{\prime}(t)^{2} d t\right)^{1 / 2} \leq C_{1} \frac{T^{3 / 2}}{12}
$$

and it immediately follows that

$$
u(t) \leq m+C_{1} \frac{T^{2}}{12}, \quad \forall t \in[0, T] .
$$

Next, let $\tau \in[0, T]$ be such that $u(\tau)=m$; multiplying (3.4) by $u^{\prime}(t)$ and integrating in $[\tau, t]$ it turns out that

$$
\begin{aligned}
\frac{u^{\prime}(t)^{2}}{2} & +\int_{\tau}^{t} H_{\epsilon}\left(u^{\prime}(s)\right) u^{\prime}(s) d s+\int_{\tau}^{t} g(u(s), \lambda) u^{\prime}(s) d s \\
& =\bar{\varphi} \int_{\tau}^{t} u^{\prime}(s) d s+\lambda \int_{\tau}^{t} \tilde{\varphi}(s) u^{\prime}(s) d s .
\end{aligned}
$$

Since $H_{\epsilon}(z) z \geq 0 \forall z \in \mathbb{R}$ we infer from this that

$$
\int_{\tau}^{t} g(u(s), \lambda) u^{\prime}(s) d s=\lambda \int_{u(\tau)}^{u(t)} g(u) d u+(1-\lambda) \int_{u(\tau)}^{u(t)} g_{0}(u) d u
$$


is bounded above. Therefore, in view of (ii), we see that there exists $\alpha>0$ (independent of $\epsilon$ and $\lambda$ ) such that

$$
u(t) \geq \alpha, \quad \forall t \in[0, T] .
$$

From (3.4) we also deduce that

$$
\int_{0}^{T} u^{\prime \prime}(t)^{2} d t \leq \int_{0}^{T}|\tilde{\varphi}(t)-g(u(t), \lambda)|\left|u^{\prime \prime}(t)\right| d t,
$$

and, since it is now known that $g(u(t), \lambda)$ is bounded independently of $\epsilon$ and $\lambda$, the same is true for $\int_{0}^{T} u^{\prime \prime}(t)^{2} d t$. Together with previous estimates, this yields the desired bound on $\|u\|_{C^{1}[0, T]}$ and the Claim is proved.

Consider the bounded, open set $\Omega:=\left\{u \in C^{1}[0, T]: \alpha<\min u<R, \max u>\right.$ $\left.r,\|u\|_{C^{1}[0, T]}<\beta\right\}$. In view of the above Claim, to see that the homotopy (3.4) has no solutions $u \in \partial \Omega$ it suffices to show that no solution $u$ of (3.4) can satisfy $\max u=r$ or $\min u=R$. The case $\max u=r$ is easily excluded by our choice of $r$. Take the case $\min u=R$ : if the first condition in (i) holds, we see in the same manner that it is impossible; if the second condition in (i) holds, then, by (3.6) we would have $u(t) \in J \forall t \in[0, T]$, and, integrating (3.4) in $[0, T]$ we reach a contradiction.

For $\lambda=0$ (3.4) has a unique (constant) solution in $\Omega$ with nonzero local index, and standard arguments of the Leray-Schauder degree theory (see [3]) allow us to conclude that (3.3) has a solution in $\Omega$ as well. This completes the proof.

Theorem 3. Let $g:] 0,+\infty[\rightarrow \mathbb{R}$ be a continuous function such that

(i) There exists $R \in \mathbb{R}$ such that either $g(R)>\varphi(t)+1, \forall t \in \mathbb{R}$, or $g(u)>\bar{\varphi}+1$ $\forall u \in] R,+\infty[$.

(ii) $\int_{0}^{1} g(u) d u=-\infty$.

(iii) There exist constants $0<\gamma<\frac{\pi^{2}}{T^{2}}$ and $C>0$ such that $g(u) \leq \gamma u+C$ $\forall u>0$.

Then the problem (3.1) admits at least one solution.

Proof. The proof is similar to the preceding one and so we omit some details. Again a homotopy (3.4) is considered, where we now make a choice of $g_{0}$ to be a $C^{1}$, strictly increasing function in $] 0,+\infty\left[\right.$ such that $g_{0}^{\prime}(+\infty)=\gamma$.

The main difference now is in the way to obtain the estimates for $u$ and $u^{\prime}$. First, multiplying (3.4) by $u$ and integrating we have

$$
\int_{0}^{T} u^{\prime}(t)^{2} d t \leq \gamma \int_{0}^{T} u(t)^{2} d t+\int_{0}^{T}(C+1+|\varphi|)|u| d t
$$

then since, as noted in [15], for all $T$-periodic functions $u \in H^{1}(0, T)$ with $m:=$ $\min u$,

$$
\int_{0}^{T}(u(t)-m)^{2} d t \leq \frac{T^{2}}{\pi^{2}} \int_{0}^{T} u^{\prime}(t)^{2} d t,
$$

we arrive at the following inequality:

$$
\|u\|_{2}^{2} \leq 2 R\|u\|_{1}+\frac{T^{2}}{\pi^{2}}\left\|u^{\prime}\right\|_{2}^{2}
$$

Since $\gamma<\frac{\pi^{2}}{T^{2}}$ we obtain a bound for $\|u\|_{2}$ and then for $\left\|u^{\prime}\right\|_{2}$. 


\section{Attractive Forces}

Theorem 4. Assume in addition to $(\mathrm{N})$ that

$$
\limsup _{u \rightarrow+\infty} g(u)<\limsup _{u \rightarrow 0^{+}} g(u)=+\infty .
$$

Then for each periodic continuous $\varphi(t)$ such that

$$
\bar{\varphi}>\limsup _{u \rightarrow+\infty} g(u)+1,
$$

the problem (3.1) has at least one solution.

Proof. We again consider the approximated problem (3.3). Let us construct lower and upper solutions for this problem. Clearly, we can select a small positive constant $a$ such that $-1+g(a) \geq \varphi(t) \forall t \in \mathbb{R}$ and this is a lower solution.

Now let us introduce the problem with a real parameter $h$

$$
z^{\prime \prime}+H_{\epsilon}\left(z^{\prime}\right)=h+\tilde{\varphi}(t), \quad z(0)=z(T), \quad z^{\prime}(0)=z^{\prime}(T) .
$$

By a theorem of Mawhin [11 we know that for fixed $\tilde{\varphi}$ there exists $h=h_{\epsilon}$ such that (4.1) is solvable. Moreover, it is easy to see that $\left|h_{\epsilon}\right| \leq 1$. Also, a solution of (4.1) is given by

$$
z_{\epsilon}(t)=\int_{0}^{t} v_{\epsilon}(s) d s
$$

where $v_{\epsilon}$ is a fixed point of the nonlinear operator

$$
v \mapsto \int_{0}^{t} V(s) d s-\frac{1}{T} \int_{0}^{t} V(s)(T-s) d s,
$$

$V$ being given by $V(s)=-H_{\epsilon}(v(s))+\frac{1}{T} \int_{0}^{T} H_{\epsilon}(v(\tau)) d \tau+\tilde{\varphi}(s)$. Note that this operator has bounded range independent of $\epsilon$.

Let $b>0$ be a number such that $u \geq b$ implies $g(u) \leq \bar{\varphi}-1$. Setting

$$
-K_{\epsilon}=\min _{t}\left(z_{\epsilon}(t)-b\right)
$$

we conclude, by (4.2) and (4.3), that the functions

$$
y_{\epsilon}(t)=z_{\epsilon}(t)+K_{\epsilon}
$$

are uniformly bounded with respect to $\epsilon$. Moreover,

$$
y_{\epsilon}^{\prime \prime}+H_{\epsilon}\left(y_{\epsilon}^{\prime}\right)+g\left(y_{\epsilon}\right)=z_{\epsilon}^{\prime \prime}+H_{\epsilon}\left(z_{\epsilon}^{\prime}\right)+g\left(y_{\epsilon}\right) \leq h_{\epsilon}+\tilde{\varphi}(t)+\bar{\varphi}-1 \leq \varphi(t),
$$

so that $y_{\epsilon}$ is the upper solution we look for. Therefore (4.1) has a solution $u_{\epsilon}$ such that

$$
a \leq u_{\epsilon}(t) \leq y_{\epsilon}(t), \quad t \in \mathbb{R} .
$$

Integrating (4.1) we infer that the family $u_{\epsilon}$ is uniformly bounded in $C^{1}$-norm, so that we can extract a subsequence $u_{\epsilon} \rightarrow u$ as $\epsilon \rightarrow 0$ and the conclusion follows as in the preceding cases.

Remark. In the course of revising the manuscript, the authors have learned that in a paper just published, Torres [14] has used the same approximation procedure to study problems similar to those that concern us here. 


\section{REFERENCES}

[1] Bonheure D. and De Coster C., Forced singular oscillators and the method of lower and upper solutions, Séminaire de Mathématique, Univ. Catholique de Louvain, Rapport n. 320, Jan. 2002.

[2] Bothe D., Periodic solutions of non-smooth friction oscillators, Z. Angew. Math. Phys. 50 (1999), 779-808. MR 2000f:34078

[3] Deimling K., Nonlinear Functional Analysis, Springer 1985. MR 86j:47001

[4] Deimling K., Resonance and Coulomb friction, Differential and Integral Equations 7 (1994), 759-765. MR 95b:34030

[5] Deimling K. and Szilágyi P., Periodic solutions of dry friction problems, Z. Angew. Math. Phys. 45, (1994), 53-60. MR 94m:34099

[6] Filippov A. F., Differential Equations with Discontinuous Righthand Sides, Kluwer Acad. Publ., Dordrecht 1988. MR 90i:34002

[7] Guenther R. B., Lee J. W. and Senkyrik, M., The Filippov approach to boundary and initial value problems and applications, Henderson, Johnny (ed.), Boundary value problems for functional differential equations, Singapore: World Scientific, 153-163 (1995). MR 96m:34034

[8] Habets P. and Sanchez L., Periodic solutions of some Liénard equations with singularities, Proc. Amer. Math. Soc. 109 (1990), 1035-1044. MR 90k:34049

[9] Kunze M., Non-smooth dynamical systems, Lecture Notes in Mathematics 1744, Springer 2000. MR 2002e:34002

[10] Lazer A. C. and Solimini S., On periodic solutions of nonlinear differential equations with singularities, Proc. Amer. Math. Soc. 99 (1987), 109-114. MR 87k:34064

[11] Mawhin J., Some remarks on semilinear problems at resonance where the nonlinearity depends only on the derivatives, Acta Math. et Informatica Univ. Ostraviensis 2 (1994), 61-69. MR 95j:34031

[12] Senkyrik, M., Periodic solutions of a second order differential equation with discontinuities in the spatial variable, Top. Methods in Nonlinear Analysis (1995), 199-206. MR 97d:34041

[13] Senkyrik, M. and Guenther, R. B., Boundary value problems with discontinuities in the spatial variable, J. Math. Anal. Applications 193 (1995), 296-305. MR 96c:34038

[14] Torres, P. J., Periodic solutions of some differential equations with nonlinear damping, Differential Integral Equations 15 (2002), 17-32.

[15] Zhang M., Periodic solutions of damped differential systems with repulsive singular forces, Proc. Amer. Math. Soc. 127 (1999), 401-407. MR 99k:34092

Departamento de Análise Matemática, Facultade de Matemáticas, Universidade de Santiago de Compostela, 15782 Santiago de Compostela, Spain

E-mail address: cabada@usc.es

Centro de Matemática e Aplicações Fundamentais, Universidade de Lisboa, Avenida Professor Gama Pinto, 2, 1649-003 Lisboa, Portugal

E-mail address: sanchez@lmc.fc.ul.pt 